\title{
BMJ Open Psychosocial interventions for disruptive behavioural problems in children living in low- and middle- income countries: study protocol of a systematic review
}

\author{
Matthew D Burkey, ${ }^{1}$ Megan Hosein, ${ }^{2}$ Marianna Purgato, ${ }^{3,4}$ Ahmad Adi, ${ }^{2}$ \\ Isabella Morton, ${ }^{2}$ Brandon A Kohrt, ${ }^{5}$ Wietse $\mathrm{A} \mathrm{Tol}^{3}$
}

To cite: Burkey MD, Hosein M, Purgato M, et al. Psychosocial interventions for disruptive behavioural problems in children living in low- and middle-income countries: study protocol of a systematic review. BMJ Open 2015;5:e007377. doi:10.1136/bmjopen-2014007377

- Prepublication history and additional material is available. To view please visit the journal (http://dx.doi.org/ 10.1136/bmjopen-2014007377)

Received 4 December 2014 Revised 23 March 2015 Accepted 25 March 2015

CrossMark

For numbered affiliations see end of article.

Correspondence to Dr Matthew Burkey; mburkey1@jhmi.edu

\section{ABSTRACT}

Introduction: Disruptive behaviour disorders (DBDs)

are among the most common forms of child psychopathology and have serious long-term academic, social, and mental health consequences worldwide. Psychosocial treatments are the first line of evidence-based treatments for DBDs, yet their effectiveness often varies according to patient sociodemographic characteristics, practice setting, and implementation procedures. While a large majority of the world's children live in low- and middle-income countries (LMIC), most studies have evaluated psychosocial treatments for DBDs in high-income Anglo countries.

Methods and analysis: The primary objective of this systematic review is to assess the effects of psychosocial treatments for DBDs in children and adolescents (under age 18) diagnosed with oppositional defiant disorder, conduct disorder, or other disruptive behavioural problems living in LMIC. The secondary objectives are to: (1) describe the range and types of psychosocial treatments used to address DBDs in LMIC and (2) identify key dissemination and implementation factors (adaptation processes, training/ supervision processes, and financial costs). All controlled trials comparing psychosocial treatments versus waiting list, no treatment, or treatment as usual in children living in LMIC will be included. Studies will be identified using the methods outlined in the Preferred Reporting Items for Systematic reviews and Meta-Analyses (PRISMA) guidelines without restrictions on language, publication type, status, or date of publication. The primary outcome measures will be disruptive behavioural problems (eg, oppositionality, defiance, aggression or deceit). Secondary outcomes will be positive mental health outcomes (eg, prosocial behaviour), function impairment, institutionalisation (or hospitalisation), academic outcomes and caregiver outcomes.

Ethics and dissemination: This study uses data from published studies; therefore ethical review is not required. Findings will be presented in a published manuscript.

\section{Strengths and limitations of this study}

- This study addresses the lack of evidence-based treatments for child mental disorders in lowresource settings through a systematic review of existing literature.

- The study uses a broad search strategy and includes databases (ie, regional databases from WHO) that are most likely to include studies from relevant geographic areas.

- The anticipated high degree of clinical and methodological heterogeneity among included studies may preclude a meta-analysis.

Trial registration number: PROSPERO CRD42014015334.

\section{INTRODUCTION}

\section{Significance}

Disruptive behaviour disorders (DBDs) are among the most common forms of child psychopathology, and have serious long-term consequences, including academic failure, development of depression, criminal conviction as adults and suicide. ${ }^{12}$ For example, oppositional defiant disorder (ODD) and conduct disorder (CD) have an estimated worldwide prevalence of $3.3 \%$ and $3.2 \%$, respectively. ${ }^{3}$ The sizeable burden of behavioural problems over the lifespan is suggested by their early age of onset, ${ }^{4}$ stability over time $^{56}$ and increased risk of onset of other psychiatric disorders throughout childhood, adolescence and adulthood, including conduct disorder (for ODD), ${ }^{7}$ depression ${ }^{8}$ and substance abuse. ${ }^{9}$ Disruptive behavioural problems are associated with low school 
achievement $^{10}$ school dropout ${ }^{11}$ and suicide. ${ }^{12}$ Long-term follow-up studies have demonstrated that childhood behavioural problems are associated with later lower educational achievement, ${ }^{13}$ increased delinquency ${ }^{14}$ and increased risk of later serious, violent and chronic criminal offending. ${ }^{15}$

The Global Burden of Disease Study 2010 estimated that mental and substance use disorders comprise the leading causes of years lived with disability, worldwide. ${ }^{16}$ Epidemiological studies have demonstrated that the majority of mental disorders have their onset in childhood or adolescence, ${ }^{4}$ drawing attention to the importance of preventive and early intervention efforts in childhood. In response, the development of locally appropriate early interventions and improvement of children's access to care in low- and middle-income countries (LMIC) have been identified as top research priorities to advancing global mental health over the upcoming decade. ${ }^{17}$

\section{Definitions}

For the purposes of this study, we refer to child behavioural problems (or 'disruptive behavioural problems') as including the diagnostic categories of "Oppositional Defiant Disorder" (ODD) and "Conduct Disorder" (CD) as specified in the Diagnostic and Statistical Manual of Mental Disorders, ${ }^{18}$ as well as symptoms of ODD and/or $\mathrm{CD}$, such as non-compliance, aggression, disruptive classroom behaviours, destroying or stealing property, and violating rules and/or laws more broadly. These problems are also commonly referred to as 'externalising' disorders. This broad scope is chosen given ongoing debates about the construct validity of current psychiatric nosology, ${ }^{19-21}$ the relationship between ODD and $\mathrm{CD}$ disorder constructs (ie, separate, overlapping or differing in degree), ${ }^{2}$ and the relative dearth of studies establishing the validity of child behaviour disorder constructs outside of North America and Western Europe. ${ }^{3}$ There is also significant overlap between the disorder constructs and aggressive and delinquent behaviours more generally, ${ }^{18}$ and studies tend to focus on disorders versus behavioural patterns depending on academic discipline (eg, psychiatry, psychology, education, sociology, criminology).

\section{Description of the intervention}

Interventions for disruptive behavioural problems have focused mostly on parent-child interactions, and are among the most well-researched treatments in child mental health in industrialised (high-income) countries. Currently available efficacy studies of treatments for disruptive behavioural problems support psychosocial treatments, such as behavioural parent training (BPTs), as the 'first-line' treatments. ${ }^{22}{ }^{23}$ BPTs are based on social learning techniques and rely heavily on operant-conditioning principles carried out through differential reinforcement of desired behaviours and consistent limit setting. ${ }^{24}$ The efficacy of BPTs for behavioural problems has been demonstrated across development, from early childhood $^{23}$ through adolescence. ${ }^{25}$

However, interventions addressing additional risk processes in the development or maintenance of disruptive behavioural problems ${ }^{26}$ have also been proposed. ${ }^{27}$ There is some suggestion that interventions that target risk processes at various levels of social ecological causation may be more effective than interventions targeting a single risk factor. ${ }^{28-30}$ With regard to approaches in LMIC, a widely used strategy for addressing mental health problems is WHO's mental health Gap Action Programme (mhGAP). ${ }^{31}$ The mhGAP Intervention Guide $^{31}$ supports the use of a variety of psychosocial interventions for child behavioural problems, including: family psychoeducation, parent skills training, coordination of academic supports, specific psychological therapies, family-based problem-solving techniques, and caregiver support. However, the current mhGAP recommendations for treatment of child behavioural problems are based on limited data from LMIC patient populations.

\section{Why it is important to do this review}

The generalisability of the available evidence for efficacy of BPTs to LMIC settings may be limited by the narrow representativeness of the study populations in the most rigorous efficacy trials, most of which were drawn from high-income (mostly English speaking) countries. For example, the 13 studies included in a Cochrane Review of behavioural treatments for early conduct disorder ${ }^{23}$ were conducted in the USA (4 in Seattle, Washington, alone), Europe or Australia with patient samples ranging from $80 \%$ to $100 \%$ Caucasian. The studies in another related Cochrane Review (for conduct disorder in ages $\left.10-17^{25}\right)$ were all conducted either in the USA $(n=7)$ or Australia $(n=1)$. The generalisability of current findings is called into question by differences between sociocultural settings in parenting practices (including disciplining styles), socioeconomic and health system characteristics, patterns of risk and protective factors, the expression of distress, and help-seeking behaviour. $^{22}$ 32-39 For example, two meta-analyses evaluating moderators of effectiveness in parent training programmes found that low family income was the strongest moderator of treatment effect size. ${ }^{33} 38$

Despite evidence of efficacy in treatment trials, response rates to psychosocial interventions for child behavioural problems remain poor for families at highest risk of long-term consequences, including those of low SES and members of marginalised cultural minority groups. ${ }^{32}$ In the setting of increasing recognition of the burden of mental disorders around the world, ${ }^{16}$ consensus is building around the priorities to: understand the role of social factors in the early development of mental disorders; develop locally appropriate early interventions; improve children's access to care in LMIC; and develop valid definitions to assess the global burden of 
mental disorders. ${ }^{17}$ However, many questions remain about how existing evidence-based treatments can be effectively utilised, and how existing local resources can be leveraged to achieve treatment success. These challenges are especially acute for child behavioural problems, where few treatments have been tested in LMIC, and where social validity concerns are magnified given the wide cultural variety of expectations related to symptoms and proposed treatments.

\section{Objectives}

The primary objective of this systematic review is to assess the effects of psychosocial treatments for DBDs in children (under age 18) diagnosed with ODD, CD or other disruptive behavioural problems living in LMIC. The secondary objectives of this study are to describe the range and types of psychosocial treatments used to address DBDs in LMIC and key issues related to implementation and dissemination. Specific secondary objectives related to implementation and dissemination include: to identify gaps in representativeness of study samples, threats of bias and generalisability of study findings; to describe currently utilised adaptation processes and training/supervision processes; and to estimate financial costs and human resource requirements of interventions.

\section{METHODS AND ANALYSIS}

\section{Types of studies}

All controlled comparison designs will be initially included except cross-over trial designs, given concerns about carry-over effects in behavioural interventions. If a sufficient number of randomised trials are identified, non-randomised studies may be dropped from further analysis.

\section{Types of participants and settings}

Children and adolescents (under age 18 years) of both sexes who have been diagnosed or screened positive, as having a disruptive behaviour disorder or moderately severe behavioural problem, will be included. We will include studies primarily addressing diagnoses of disruptive behaviour-related disorders as outlined in the Diagnostic and Statistical Manual (editions III through 5), ${ }^{18} 4041$ including: Oppositional Defiant Disorder (or Oppositional Disorder in DSM-III), CD, and Disruptive Behavior Disorder Not Otherwise Specified. We will also include diagnoses of CD (F91) and its subcategories from the International Classification of Disease (all editions). ${ }^{42}$ We will also include other related disruptive behavioural problems diagnosed by the use of a previously validated diagnostic or screening instrument primarily addressing problems related to behaviours considered disruptive, oppositional, defiant, or otherwise rule-breaking. We will exclude studies primarily addressing Attention Deficit/Hyperactivity Disorder or other mental disorders. We will include studies conducted among persons living in LMIC, as defined by the World Bank. ${ }^{43}$ We will include studies conducted in any practice setting, including, but not limited to: clinics, schools, community, hospitals and juvenile justice systems. We will exclude studies conducted in highincome countries.

\section{Types of interventions}

Interventions will include any 'active' psychosocial treatment, including, but not limited to: individual, family, or group therapies; education; training; or guidance that primarily targets: children, parents, families, or teachers. We will also include system-wide interventions targeting ecological risk factors, including, but not limited to: school policies, neighbourhood or community factors, and family economic interventions, providing that they meet the other inclusion criteria. Comparison groups may include: no treatment, wait list controls, 'treatment as usual' groups, or 'inactive controls'.

\section{Types of outcome measures \\ Primary outcomes}

Disruptive behavioural problems/symptoms (measured with the Child Behavior Checklist, ${ }^{44}$ Strengths and Difficulties Questionnaire, ${ }^{45}$ or other validated scales).

\section{Secondary outcomes}

- Positive mental health outcomes (eg, prosocial behaviours).

- Functioning (measured with Child Function Impairment Measure ${ }^{46}$ or with any other commonly used measure).

- (Juvenile) justice contact (number of arrests, incarcerations, or other contacts with juvenile justice authorities).

- Institutionalisation (number of days in an institutional setting).

- Hospitalisation (number of hospitalisations or number of days in hospital).

- Academic outcomes (number of school absences, suspensions or expulsions).

- Parental distress or mental health (measured with the Parenting Stress Index, ${ }^{47}$ General Health Questionnaire $^{48}$ or other commonly used measures).

- Parenting skills, attitudes or behaviours (including discipline patterns and abuse towards child; measured with the Home Observation for Measurement of the Environment, ${ }^{49}$ or other commonly used measures).

- Loss to follow-up.

\section{Timing of outcome assessment}

Measurements at any time point will be eligible for inclusion. In the case of assessment at multiple time points, the last assessment for which comparative data is available will be used. 


\section{Search methods for identification of studies}

The search strategy will be developed in consultation with information specialists at the Johns Hopkins Medical Institutions Welch Library (see online supplementary appendix S1 for a preliminary search strategy for MEDLINE). We will search the following electronic databases for primary studies: MEDLINE, PsycINFO, CENTRAL and the World Health Organization Global Health Library regional indexes (AIM (AFRRO), IMEMR (EMRO), IMSEAR (SEARO, WPRIM, WPRO)). We will also search the reference lists from articles of interest, including previous systematic reviews on related topics. We will also identify ongoing or incomplete studies by searching the metaRegister of Controlled Trials. We will not exclude studies based on language of publication (translation will be sought).

\section{Data collection and analysis}

Selection of studies

Two authors will independently screen the search results (titles and abstracts) for eligibility. For studies identified as possibly eligible by either of the reviewers, we will search for the full-text article. Two authors will then review the full-text articles for final inclusion based on the study inclusion/exclusion criteria. Reasons for exclusion will be noted for each excluded study. Disagreements will be resolved through discussion with a third author.

\section{Data extraction and management}

Two study authors will independently review the full-text articles and extract relevant data fields using a structured, standardised online data collection form. Prior to data collection, we will pilot the form on at least four included studies. Disagreements will be resolved by discussion between the study authors; if the initial reviewers do not achieve consensus, a third author will resolve the disagreement. We will contact the corresponding authors of included articles to clarify questions, and request additional data not included in the original publication. We will extract the following data from each included study:

- Geographic setting (country(ies), rural/urban)

- Reported study type (eg, RCT, quasiexperimental, etc)

- Participant allocation processes (using the standardised tool from the Cochrane handbook, Table 13.2. ${ }^{50}$ )

- Practice setting (eg, primary care clinic, community, school, etc)

- Patient inclusion/exclusion criteria (diagnosis, etc)

- Patient characteristics (age range, \%female, socioeconomic status indicators)

- Intervention description (reference, content, targets, duration)

- Intervention adaptations (from what protocol? adaptations to content, adaptations to mode of delivery)

- Implementer characteristics (qualifications, training, supervision)

- Control arm treatment description

- Primary outcome measure (scale, subscale, etc)
- Secondary outcome measures

- Number randomised (by arm)

- Follow-up rate and handling of dropouts

- Estimate of effect size and precision (eg, OR, RR and $\mathrm{CI}, \mathrm{p}$ value; univariate and multivariate)

- Adverse effects reported (by arm)

- Follow-up period

- Funding source

\section{Assessment of risk of bias in included studies}

Two study authors will independently assess the risk of bias in each of the included studies using the Cochrane Collaboration's tool for assessing risk of bias. ${ }^{51}$ Disagreements will be resolved by discussion between the study authors, or arbitration by a third author, if necessary. This tool identifies key domains through which bias is likely to be introduced into trial design, conduct or analysis: selection bias, performance bias, detection bias, attrition bias, reporting bias and other biases. For each domain, the tool identifies potential sources of bias, prompts the reviewer to provide support for a judgement, and requests the review author's judgement concerning the level of bias (low, unclear or high risk of bias).

\section{Data analysis}

Measures of treatment effect

We will first calculate the estimated treatment effect in each study using a standardised mean difference and its SD for continuous data, and OR with its $95 \%$ CI for dichotomous data. For statistically significant results, we will calculate the number needed to treat to provide benefit, and the number needed to treat to induce harm. If possible, we will perform a meta-analysis including similar studies based on target population, intervention design and outcome assessment. Meta-analysis is planned for the primary and secondary outcomes, according to their availability. All statistical tests will be performed using Stata. ${ }^{52}$

\section{Unit of analysis issues}

Given the relevance of cluster randomisation designs to the topic of interest (eg, interventions at the school or community level), cluster-randomised trials will be eligible for inclusion if they meet other inclusion criteria. Preference will be given to analyses conducted with the cluster as the unit of analysis. Sensitivity analyses will be conducted to assess the influence of including clusterrandomised trials in the overall effect estimate. Cross-over trials will not be included in this study given the high likelihood of carry-over effects of behavioural interventions. For multiarm treatment studies, all treatment arms that individually meet criteria for inclusion will be included if they are eligible for pair-wise comparison criteria.

\section{Dealing with missing data}

Dropouts will be dealt with on an intention-to-treat (ITT) basis if data is available. If ITT analysis is not 
available, as-treated analysis will be included. Missing precision estimates (CIs or SDs) that we are not able to obtain from the authors will be dealt with by assuming the highest SD from within the group of studies under analysis.

\section{Assessment of reporting biases}

We will assess the possibility of publication bias using funnel plots.

\section{Data synthesis}

We will present both narrative and quantitative (ie, tabular and meta-analysis, if possible) syntheses of the data, focusing on questions related to the primary and secondary objectives. For all analyses, randomised and non-randomised trials will be presented separately. Non-randomised trials will not be included in the meta-analyses. The narrative synthesis will describe the design, adaptation and implementation aspects of the studies, whereas, quantitative syntheses will elucidate the patient and provider characteristics, and primary and secondary outcomes. We will provide summary statistics describing patient and provider (sociodemographic) characteristics for each study and for all studies pooled together. We will include forest plots summarising the results of individual studies and the meta-analyses.

\section{Subgroup analysis and assessment of heterogeneity}

Heterogeneity will be assessed using $\chi^{2}$ tests for homogeneity (using $\mathrm{p}<0.10$ as a cut-off) and the $\mathrm{I}^{2}$ statistic. We will explore potential sources of heterogeneity by considering clinical and methodological aspects of diversity. We will address the anticipated heterogeneity by using random effects meta-analysis models. In addition, if there are a sufficient number of included studies, we will conduct subgroup analyses based on the following characteristics: primary diagnosis (ie, ODD vs CD); individual or family versus school or community interventions; specialist versus non-specialist implementers.

\section{Sensitivity analysis}

We will conduct sensitivity analyses to assess the influence of design decisions in the systematic review (eg, eligibility criteria, which data to include, and analysis methods, as identified throughout the implementation of the review, as recommended by the Cochrane Handbook (Section $9.7)^{50}$ on the results of the meta-analysis. We plan to conduct a sensitivity analysis including and excluding cluster-randomised trials. We also plan to assess the influence of individual studies by using the 'leave-one-out' sensitivity method using the metainf command in Stata.

\section{Presentation of results and summary of findings table}

We will tabulate the sources and flow of studies throughout the review process, including reasons for exclusion, as outlined in the Preferred Reporting Items for Systematic reviews and Meta-Analyses statement. ${ }^{53}$ We will present our key findings in a 'summary of findings' table using the Grading of Recommendations, Assessment, Development and Evaluation (GRADE) approach, ${ }^{54}$ addressing the quality of evidence, estimated effect sizes, and the secondary objectives outlined above ${ }^{55}{ }^{56}$ We will also record any departures from the standard methods described in Chapter 11 and Chapter 12 of the Cochrane Handbook, ${ }^{50}$ along with a justification for such departures.

\section{Ethics and dissemination \\ Ethical issues}

The planned study relies on previously conducted studies and does not involve collection of new or identifiable data. Accordingly, no ethical review or informed consent is required.

\section{Publication plan}

This study has been registered in the International Prospective Register of Systematic Reviews (http://www. crd.york.ac.uk/prospero) (registration number: CRD420 14015334). The authors plan to present the findings of this review in a single published manuscript.

\section{Author affiliations}

${ }^{1}$ Division of Child and Adolescent Psychiatry, Johns Hopkins School of Medicine, Baltimore, Maryland, USA

${ }^{2}$ Johns Hopkins Bloomberg School of Public Health, Baltimore, Maryland, USA

${ }^{3}$ Department of Mental Health, Johns Hopkins Bloomberg School of Public Health, Baltimore, Maryland, USA

${ }^{4}$ Section of Psychiatry, World Health Organization Collaborating Centre for Research and Training in Mental Health and Service Evaluation, University of Verona, Verona, Italy

${ }^{5}$ Duke University Global Health Institute, Durham, North Carolina, USA

Twitter Follow Wietse Tol at @wietsetol

Acknowledgements The authors wish to thank Donna Hesson for her valuable feedback in preparing the search strategy.

Contributors MDB, WAT and MH conceived the study and drafted the initial protocol. MP, AA, IM and BAK assisted in protocol design. MDB and MP reviewed the statistical analysis plan. All authors read and approved the final manuscript.

Funding This work was supported by the Johns Hopkins Clinical Research Scholar Award (MDB) grant number 5KL2TR001077-02. This article was funded by the National Institutes of Health in the U.S.

Competing interests None declared.

Provenance and peer review Not commissioned; externally peer reviewed.

Data sharing statement Additional search strategies are available from the corresponding author upon request.

Open Access This is an Open Access article distributed in accordance with the Creative Commons Attribution Non Commercial (CC BY-NC 4.0) license, which permits others to distribute, remix, adapt, build upon this work noncommercially, and license their derivative works on different terms, provided the original work is properly cited and the use is non-commercial. See: http:// creativecommons.org/licenses/by-nc/4.0/

\section{REFERENCES}

1. Loeber R, Burke JD, Lahey BB, et al. Oppositional defiant and conduct disorder: a review of the past 10 years, part I. J Am Acad Child Adolesc Psychiatry 2000;39:1468-84. 
2. Burke JD, Loeber R, Birmaher B. Oppositional defiant disorder and conduct disorder: a review of the past 10 years, part II. J Am Acad Child Adolesc Psychiatry 2002;41:1275-93.

3. Canino G, Polanczyk G, Bauermeister JJ, et al. Does the prevalence of $\mathrm{CD}$ and ODD vary across cultures? Soc Psychiatry Psychiatr Epidemiol 2010;45:695-704.

4. Kessler RC, Angermeyer M, Anthony JC, et al. Lifetime prevalence and age-of-onset distributions of mental disorders in the World Health Organization's World Mental Health Survey Initiative. World Psychiatry 2007:6:168.

5. Olweus D. Stability of aggressive reaction patterns in males: a review. Psychol Bull 1979;86:852.

6. Stattin $\mathrm{H}$, Magnusson $\mathrm{D}$. Stability and change in criminal behaviour up to age 30. Br J Criminol 1991;31:327-46.

7. Cohen P, Flory M. Issues in the disruptive behavior disorders: attention deficit disorder without hyperactivity and the differential validity of oppositional defiant and conduct disorders. DSM-IV Sourcebook 1998;4:455-63.

8. Burke JD, Hipwell AE, Loeber R. Dimensions of oppositional defiant disorder as predictors of depression and conduct disorder in preadolescent girls. J Am Acad Child Adolesc Psychiatry 2010;49:484-92.

9. Boyle $\mathrm{MH}$, Offord DR. Psychiatric disorder and substance use in adolescence. Can J Psychiatry 1991;36:699-705.

10. Olweus D. Low school achievement and aggressive behavior in adolescent boys. In: Magnusson D, Allen VL, eds. Human development: an interactional perspective. New York, NY: Academic Press, 1983:353-65.

11. Tramontina S, Martins S, Michalowski MB, et al. School dropout and conduct disorder in Brazilian elementary school students. Can $J$ Psychiatry 2001;46:941-7.

12. Nock MK, Borges G, Bromet EJ, et al. Cross-national prevalence and risk factors for suicidal ideation, plans and attempts. $\mathrm{Br} \mathrm{J}$ Psychiatry 2008;192:98-105.

13. Huesmann LR, Eron LD, Yarmel PW. Intellectual functioning and aggression. J Pers Soc Psychol 1987;52:232.

14. Tremblay RE, Masse B, Perron D, et al. Early disruptive behavior, poor school achievement, delinquent behavior, and delinquent personality: Iongitudinal analyses. J Consult Clin Psychol 1992;60:64.

15. Loeber R, Farrington DP. Young children who commit crime: Epidemiology, developmental origins, risk factors, early interventions, and policy implications. Dev Psychopathol 2000;12:737-62.

16. Whiteford HA, Degenhardt L, Rehm J, et al. Global burden of disease attributable to mental and substance use disorders: findings from the Global Burden of Disease Study 2010. Lancet 2013;382:1575-86.

17. Collins PY, Patel V, Joestl SS, et al. Grand challenges in global mental health. Nature 2011;475:27-30.

18. American Psychiatric Association. Diagnostic and statistical manual of mental disorders, DSM-5. Arlington, VA, 2013.

19. Insel TR. Directors blog: transforming diagnosis. National Institute of Mental Health, 2013 (cited 3 Jan 2014). http://www.nimh.nih.gov/ about/director/2013/transforming-diagnosis.shtml

20. McHugh PR. Striving for coherence. JAMA 2005;293:2526-8.

21. Gaines AD. From DSM-I to III-R; Voices of self, mastery and the other: a cultural constructivist reading of US Psychiatric classification. Soc Sci Med 1992:35:3-24.

22. Eyberg SM, Nelson MM, Boggs SR. Evidence-based psychosocia treatments for children and adolescents with disruptive behavior $J$ Clin Child Adolesc Psychol 2008;37:215-37.

23. Furlong M, McGilloway S, Bywater T, et al. Behavioural and cognitive-behavioural group-based parenting programmes for early-onset conduct problems in children aged 3 to 12 years. Wiley Online Library, 2012.

24. Patterson GR. Etiology and treatment of child and adolescent antisocial behavior. Behav Analyst Today 2002;3:133-45.

25. Woolfenden S, Williams K, Peat J. Family and parenting interventions in children and adolescents with conduct disorder and delinquency aged 10-17. Cochrane Database Syst Rev 2001;(2):CD003015.

26. Patterson GR, DeBaryshe BD, Ramsey E. A developmental perspective on antisocial behavior. Am Psychol 1989;44:329.

27. Lau AS, Fung JJ, Yung V. Group parent training with immigrant Chinese families: enhancing engagement and augmenting skills training. J Clin Psychol 2010;66:880-94.

28. Miller GE, Prinz RJ. Enhancement of social learning family interventions for childhood conduct disorder. Psychol Bull 1990;108:291.

29. Borduin CM, Mann BJ, Cone LT, et al. Multisystemic treatment of serious juvenile offenders: long-term prevention of criminality and violence. J Consult Clin Psychol 1995;63:569.
30. Tol WA, Song S, Jordans MJ. Annual research review: resilience and mental health in children and adolescents living in areas of armed conflict-a systematic review of findings in low- and middle-income countries. J Child Psychol Psychiatry 2013:54:445-60.

31. World Health Organization: Mental Health Gap Action Programme. MhGAP intervention guide for mental, neurological and substance use disorders in non-specialized health settings. Version 1.0. World Health Organization, 2010.

32. Kazdin AE. Parent management training: evidence, outcomes, and issues. J Am Acad Child Adolesc Psychiatry 1997;36:1349-56.

33. Lundahl B, Risser HJ, Lovejoy MC. A meta-analysis of paren training: moderators and follow-up effects. Clin Psychol Rev 2006;26:86-104.

34. Maliken AC, Katz LF. Exploring the impact of parental psychopathology and emotion regulation on evidence-based parenting interventions: a transdiagnostic approach to improving treatment effectiveness. Clin Child Fam Psychol Rev 2013;16:1-14.

35. Holden GW, Lavigne VV, Cameron AM. Probing the continuum of effectiveness in parent training: characteristics of parents and preschoolers. J Clin Child Psychol 1990;19:2-8.

36. Orrell-Valente JK, Pinderhughes EE, Valente E Jr, et al. If it's offered, will they come? Influences on parents' participation in a community-based conduct problems prevention program. Am J Community Psychol 1999;27:753-83.

37. Reid MJ, Webster-Stratton C, Beauchaine TP. Parent training in head start: a comparison of program response among African American, Asian American, Caucasian, and Hispanic mothers. Prev Sci 2001;2:209-27.

38. Reyno SM, McGrath PJ. Predictors of parent training efficacy for child externalizing behavior problems-a meta-analytic review. $J$ Child Psychol Psychiatry 2006;47:99-111.

39. Lansford JE, Chang L, Dodge KA, et al. Physical discipline and children's adjustment: cultural normativeness as a moderator. Child Dev 2005;76:1234-46.

40. American Psychiatric Association. Diagnostic and statistical manual of mental disorders, DSM-III. Washington, DC: American Psychiatric Pub Inc, 1980.

41. American Psychiatric Association. Diagnostic and statistical manual of mental disorders, DSM-IV. Washington, DC, 1994.

42. World Health Organization. International classification of disease (ICD-10). Geneva, 2010.

43. Country and Lending Groups [Internet]. 2014. http://data.worldbank. org/about/country-and-lending-groups

44. Achenbach TM, Edelbrock C. Manual for Child Behavior Checklist. Burlington, VT: University of Vermont, Department of Psychiatry, 1991.

45. Goodman R. The strengths and difficulties questionnaire: a research note. J Child Psychol Psychiatry 1997;38:581-6.

46. Tol WA, Komproe IH, Jordans MJ, et al. Developing a function impairment measure for children affected by political violence: a mixed methods approach in Indonesia. Int J Qual Health Care 2011;23:375-83.

47. Abidin RR. Parenting Stress Index: A measure of the parent-child system. 1997

48. Goldberg D, Williams P. General health questionnaire (GHQ). Swindon, Wiltshire, UK: nferNelson, 2000.

49. Caldwell BM, Bradley $\mathrm{RH}$. Home observation for measurement of the environment. University of Arkansas at Little Rock Little Rock, 1984.

50. Higgins J, Green S. eds. Cochrane handbook for systematic reviews of interventions. Version 5.1.0 ed. The Cochrane Collaboration, 2011.

51. Higgins J, Altman DG, Gøtzsche PC, et al. The Cochrane Collaboration's tool for assessing risk of bias in randomised trials. BMJ 2011:343:d5928.

52. Stata Corporation. Stata Statistical Software Release 12.0: Programming. Stata Corporation, 1985-2013.

53. Moher D, Liberati A, Tetzlaff J, et al. Preferred reporting items for systematic reviews and meta-analyses: the PRISMA statement. Ann Intern Med 2009;151:264-9.

54. Guyatt G, Oxman AD, Akl EA, et al. GRADE guidelines: 1. Introduction-GRADE evidence profiles and summary of findings tables. J Clin Epidemiol 2011:64:383-94.

55. Langendam MW, Akl EA, Dahm P, et al. Assessing and presenting summaries of evidence in Cochrane Reviews. Syst Rev 2013;2:81.

56. Barbui $\mathrm{C}$, Dua $\mathrm{T}$, van Ommeren $\mathrm{M}$, et al. Challenges in developing evidence-based recommendations using the GRADE approach: the case of mental, neurological, and substance use disorders. PLoS Med 2010;7:e1000322 